\title{
En torno al cambio cultural en la Prehistoria
}

ANA M. ${ }^{a}$ MUÑOZ AMILIBIA

El libro que motiva estas páginas es una recopilación, en lengua catalana, de las ponencias presentadas por varios autores al Coloquio celebrado en Barcelona, bajo el mismo título, en 1989, asi como las intervenciones y debate en torno a ellas ${ }^{1}$. En realidad, cómo se señala en la introducción, es el resultado de un proceso de debate teórico dirigido a poner de manifiesto la "falsa estabilidad» de la arqueología catalana y española, y al mismo tiempo tratando de suplir una falta de este tipo de planteamientos en el ámbito académico.

Las ideas expresadas en la introducción son del mayor interés, y creo que merecen un comentario antes de pasar a ver cada una de las ponencias en concreto. No sé de donde parte la idea de una arqueología estable. Cómo ciencia histórica, es lógico que vaya evolucionando en relación a dos aspectos principales: el enriquecimiento de sus propios conocimientos, y los cambios continuos a que está sometida la sociedad en que está inmersa. $Y$ este es un fenómeno irreversible. Por ello no puede hablarse de falsa estabilidad, si no más bien, no se puede pretender que tenga estabilidad, ya que, por el contrario, debe de seguir cambiando, evolucionando, cómo la propia dinámica de la sociedad. No se puede aspirar a encontrar fórmulas definitivas, aunque si adecuarlas a los nuevos niveles de conocimiento de la ciencia, tanto desde un punto de vista teórico como práctico, con la seguridad de que los que lleguen después, adoptarán de nuevo una actitud crítica y renovadora.

Josep Anfruns y Elisenda Llobet (eds.): El canvi cultural a la Prehistória. Barcelona, Columna, 1990, 358 págs. con 10 figuras. 
No deja de ser preocupante la afirmación, sin duda sincera y probablemente cierta, de las deficiencias de su enseñanza en la Universidad. Tanto en la falta de planteamientos teóricos, cómo en la formación objetiva, que se califica de superficial. Al parecer los sectores «menos instalados" son más sensibles a esta problemática, que comparten con el estamento estudiantil. Podríamos decir que hay una falta de respuesta a sus demandas, como consecuencia de enfoques metodológicos y mentalidades diferentes. $Y$ esto es lógico, en función de la formación y del contexto en que cada uno la ha recibido, dentro de un proceso al que tampoco podrán sustraerse, de forma más o menos acusada, según su propia idiosincrasia, los futuros «instalados».

En el fondo la situación refleja y responde a un fenómeno de cambio y crisis actuales - que en España se ha producido con más de diez años de retraso en relación a otros países-, que bien merecería un análisis objetivo, siguiendo las mismas pautas aplicadas por los autores del libro a los cambios de las sociedades prehistóricas. $Y$ sobre todo responder a la pregunta de porqué se ha llegado a ésta situación en cierto modo antagónica.

Seria pretencioso por mi parte entrar ahora en la problemática de la evolución social en los treinta últimos años, pero voy a intentar señalar algunos aspectos de la enseñanza de la Arqueología en la Universidad, tema que siempre me ha preocupado, tratando de buscar algunas causas que han desembocado en la situación actual.

Los principales cambios en los estudios universitarios a partir de los años sesenta han seguido una marcada tendencia a la especialización, tendencia que también incidía en la orientación de la enseñanza media de Bachillerato. Ello ha supuesto que los estudios de Humanidades se hayan ido desmembrando cada vez más hacia especialidades concretas desde el primer curso de los estudios universitarios: Filosofía, Filología, Historia, Literatura..., e incluso la Historia se ha ido compartimentando cronológicamente, restando una visión global de su devenir. Quedaban así muy menguadas las posibilidades de una formación integral, en la que el pensamiento y el diálogo intelectual, facilitaran un bagaje suficiente para comprensión del hombre y su historia.

Por un lado, el creciente aumento del conocimiento científico, obligaba a una especialización que permitiera profundizar mejor en campos concretos, pero por otro, se recortaban parcelas fundamentales para la formación intelectual del futuro especialista. En el caso de la Arqueología, en aquellos días había una enorme preocupación por actualizar las técnicas de investigación de campo y laboratorio, mientras que los proble- 
mas metodológicos quedaron relegados a otros campos de la Historia, a la Filosofia o a la Antropología. En la Universidad de Barcelona, el llamado “Plan Maluquer» quiso subsanar estas dificultades mediante un sistema sumamente abierto de asignaturas optativas, que permitieran, mediante orientaciones de tutoria, completar los elementos cardinales de una especialidad.

El problema era y es difícil. ¿El objetivo central de una especialidad ha quedado reducido a una serie de conocimientos superficiales? ¿Faltan planteamientoe teóricos que den base y sentido a un determinado campo científico? Seguramente sí. Por ello pienso que es importante y positivo que un grupo de arqueólogos se reúna para crear un debate teórico. Seria deseable que éste debate no quedara fuera del ámbito universitario, y que estuviera siempre abierto a futuros planteamientos. Lo que si parece razonable, es que, para que sea eficaz, tenga una base de conocimiento en que apoyarse. Por ello, en lo que se refiere concretamente a la Prehistoria, hay que evitar una dicotomia entre el planteamiento metodológico y el análisis arqueológico. El prehistoriador ha de ser al mismo tiempo arqueólogo e historiador, de lo contrario no estará en condiciones de ejercer una verdadera crítica y construcción científica.

Las ponencias se introducen con la de Christopher TILLEY, sobre: "Constituint una arqueología social: Un projecte modernista" (págs. 1744). Sin duda esta, como todas las demás, necesitarian de un comentario más profundo que el que voy a hacer, y que excederia los límites de una reseña. Tilley nos plantea nada menos que el problema de la relación de la arqueología con las tendencias intelectuales y culturales del Occidente contemporáneo, que se reúnen bajo el calificativo de modernista. La evolución del pensamiento intelectual ha llegado a un grado de complejidad que es muy difícil encasillarlo bajo un termino que tiene muchas connotaciones desde finales del siglo pasado, precisamente cómo reacción contra la corriente historicista, y manifiesta en todos los campos de la creación humana. Desde el arte y la literatura a la filosofía y teología, dentro de un contexto de renovación política, social y económica, en que la producción racionalizada con el creciente desarrollo industrial, constituía el signo más relevante de los tiempos nuevos. Esta corriente, vino a ser en el fondo una culminación del romanticismo en contraposición al realismo, y estuvo fuertemente influida por las tendencias de evasión sociológica en un mundo tremendamente racionalizado. De ahí sus profundas contradiciones, que en último término revelan una profunda crisis social e intelectual.

El autor concreta el sentido genérico que da al término «modernidad", como una discontinuidad temporal, una ruptura con la tradición y 
una conciencia de cambio continuo. Pienso que la ruptura con la tradición actualmente es muy difícil de establecer, ya que al punto que hemos llegado, no podemos referirnos a una sola tradición, sino más bien a una suma de experiencias muy diversas que han comportado la realidad actual, de forma que lo que hasta hace poco podia considerarse como una reacción hacia lo tradicional, queda también cuestionado y convertido en una nueva forma de integrismo. Ni siquiera parece vigente aquello de «acabar con el orden establecido». Por ello sin duda, el autor precisa esta ruptura con la tradición como «un sentimiento de novedad y sensibilidad hacia una naturaleza efímera, fugaz y contingente del presente». En este sentido, lamento la poca atención que se presta en todos estos debates a nuestros pensadores españoles, que, desde Unamuno a Ortega y Zubiri tan bien reflejaron ésta actitud crítica y vitalista, desde orientaciones intelectuales muy diversas. Sin duda no sería necesario recurrir a Marx para subrayar la consciencia del cambio.

Pero también se refiere el autor al aspecto positivo de la modernidad, con un sentimiento posibilista del cambio, como fuerza que estimula la creatividad y al mismo tiempo ahoga. Señala las contradicciones de ésta modernidad, que por un lado está inmersa en el mundo cultural del capitalismo y por otro ha sido germen del marxismo con su aspiración de cambio.

En este contexto de transformación social, desarrollo, innovación y crecimiento, analiza el tema del Coloquio, el cambio cultural en la Prehistoria, intentando comprender nuestras propias experiencias. Desde su punto de vista, la dinámica modernista ha sido insuficiente dentro de la arqueología, lo que se reflejaría en la falta de diversidad en los textos arqueológicos, fieles siempre a los mismos esquemas. Así, la explicación del cambio en el registro arqueológico, mediante una serie de procesos como la presión demográfica o los cambios ambientales y tecnológicos.

La arqueología no es algo compacto, sino fragmentaria y creadora de un nuevo espacio de identidad que no puede ser totalizador. Por ello el desarrollo de variadas actitudes nuevas, a veces contradictorias, lo califica de «una comprensión más fresca del pasado». "El desarrollo de una pluralidad de aproximaciones al pasado puede estimular de manera positiva una forma de productividad inacabable y de dinamismo", que conducirian al debate y nuevo conocimiento. Desde este punto de vista, todas las actitudes tendrian una razón de ser, incluso la nihilista. Llega a afirmar que aceptar la modernidad supone que la arqueología no es sólo una forma de trabajo, sino una forma de vida. 
Sin embargo, su crítica al historicismo, cómo la construcción de un código orientado históricamente, es claramente excluyente, aunque no quiera decir "que la historia no tenga valor, sino que tiende a ordenar los hechos de una manera 'muy particular'». Pero reconoce que "afortunadamente" en arqueología se conocen pocos hechos, por lo que no hay más remedio que centrarse en procesos generales de cambio, más allá de los actores sociales individuales. Esto es importante desde su perspectiva marxista, ya que hay que huir del concepto del individuo como motor dentro de una sociedad. Pero también critica, dentro del historicismo, la noción de proceso continuado de desarrollo cómo explicación del cambio, ya que daria lugar a una "sobrevaloración anacrónica de dinamismo y cambio". Sus argumentaciones no parecen muy sólidas, sobre todo cuando pretende paralelizarlas con nuestras sociedades modernistas "calientes", ya que, cómo él mismo indica, la historia tiene tendencia a ser acumulativa, y es lógico que ésta progresión acumulativa acelere el proceso de cambio en los tiempos actuales. Esto no parece demostrativo de que no existiera un proceso de cambio, aunque su dinámica fuera más lenta e ignoremos en muchos casos cual fuera el motor del cambio, si es que lo hubo.

Estoy de acuerdo en que estabilidad y cambio son términos muy relativos, que pienso sería interesante analizar en sociedades más recientes, buscando la posibilidad de establecer modelos teóricos que fueran demostrables. Esto no quiere decir que se apliquen a las sociedades prehistóricas, cómo se ha hecho con frecuencia y no sólo en las explicaciones de tipo historicista. Modelos que inicialmente se plantean como teóricos, pero que al aplicarlos a los datos arqueológicos se fuerzan de manera evidente, cayendo en una nueva forma de historicismo, aunque esos modelos se hayan establecido a partir de datos antropológicos o etnoarqueológicos

Es curioso que, aunque el autor critique las aproximaciones historicistas cómo simple "producción del pasado a imagen del presente", sugiera más adelante el configurar una panorámica del pasado cómo un proyecto del presente: "El pasado requiere la infusión de nuestra subjetividad, sin la cual no puede existir". Afirmación sin duda cierta, pues parece evidente que para un historiador es imposible una actitud pasiva ante el hecho arqueológico.

Aquí se plantea el problema de la objetividad y la subjetividad ante el hecho arqueológico. La objetividad seria particular y contingente. El dato arqueológico es parte de un pasado real, pero con su descubrimiento se convierte en parte de nuestro presente, $y$, desde éste punto de vista, la materialización del pasado representaria un agente objetivo, 
mientras que la del presente nos convierte en agentes subjetivos, lo que, según el autor, no supone una oposición radical entre subjetividad y objetividad, sino que ambas están estrechamente unidas. Sería necesario prescindir de epistemologías abstractas que inciden en la dicotomia absoluta entre sujeto y objeto, ya que «la experiencia arqueológica se introduce en la existencia de un mundo nuevo a través de la experiencia autobiográfica".

Considera que, en la investigación del cambio cultural en la prehistoria, el estructuralismo y la semiótica pueden proporcionar una base de análisis que permita la comprensión total del fenómeno, más allá del simple análisis empírico, descubriendo estructuras subyacentes de caracter social, planteando la incidencia de la cultura en la mediatización de la naturaleza y de la sociedad sobre el individuo. La cultura material es parte del medio en que se convierte la realidad social, y hay que considerarla cómo un elemento activo dentro de la sociedad y no cómo un simple reflejo pasivo del proceso social. Aunque el estructuralismo y la semiótica pueden ofrecer un punto de partida en el análisis de la cultura material, es importante, a juicio del autor, considerar las distintas orientaciones de la crítica post-estructuralista, en su intento de unir la teoría de la estructura con la de la acción social (poder, ideologia, práxis material y simbolismo), que enriquecerian las posibilidades de comprensión total. De todas formas, nunca habrá una interpretación ni respuesta definitivas al porqué se produce el cambio cultural. Una interpretación histórica seria exige reconocer la importancia de la discontinuidad y el cambio a lo largo del tiempo.

Finalmente, el autor reflexiona sobre la arqueología en el contexto sociopolítico actual, insistiendo en la necesidad de una estructura teórica destotalizadora, en la que un auténtico pluralismo ejerza una crítica precisa de los "diferentes pasados". Desde su punto de vista, la arqueología "debería ayudarnos a comprender y a cambiar el presente, insertándolo en una nueva relación con el pasado". Sería en el fondo una razón de ser, una justificación de la arqueología, más allá de una labor de investigación histórica aislada. En base al análisis de Gramsci sobre la negemonia, y una mejor comprensión de la cultura y educación, formula la pregunta ¿Qué es la arqueología si no tiene un objetivo educativo? Su proyección en el ámbito social, llevaría a la arqueología a una interpretación politica efectiva en el presente con una actitud crítica.

José Antonio DuEÑAS JIMÉNEZ, trata de «Les teories externalistes enfront del culturalisme en el cambi cultural» (págs. 45-62). Tras enumerar las teorias adaptativas en relación con el cambio cultural en la Prehistoria, desarrolladas en los últimos veinticinco años, y las criticas a los 
modelos por ellas establecidas, sobre todo por parte de las corrientes metodológicas individualistas y simbólicas, comenta el modelo morfogenético aplicado por Renfrew contra los procesos difusionistas en el espacio, y concretamente en la explicación del cambio al neolítico y la adopción de la metalurgia del cobre en Europa, asi cómo la teoría de las catástrofes y la dinámica del colapso sistemático, en relación al grado de centralización, de poder jerárquico y estratificación social.

La aplicación de la informática a la arqueologia y concretamente el sistema DAI (Inteligencia artificial distribuida), también es objeto de un análisis crítico. El método parte de la relación de los vestigios materiales y la ideologia, en la que el núcleo del comportamiento del sistema sociocultural reside en el intercambio y la tecnología. Como señala el autor, la primera critica al sistema es que se pretenda dar una respuesta al comportamiento humano a través del comportamiento informático, si tener en cuenta que el computador no puede abstraer procesos mentales, sino sólo reproducirlos.

Después de desarrollar las diferentes posiciones ante el proceso de adaptación, evolución y cambio cultural, concluye sus propias reflexiones sobre el problema, que, a primera vista, dada su posición crítica, pueden parecer tremendamente demoledoras. Sin embargo, afirma la necesidad de buscar metodologías que permitan aproximarnos a la realidad social, con tal de hacerlo de forma ordenada y sistemática y dentro de una praxis de contrastaciones, aunque en último término sea la ideología la que matizará ésta aproximación.

Se plantea y responde a una serie de preguntas en torno a las dificultades que presentan determinados enfoques metodológicos, cuestionando finalmente si hay que desarrollar métodos que nos permitan responder a las preguntas "que significa" o "cómo era", para poder llegar al "porqué sucedió". Esta última pregunta no tendría sentido, según el autor, si no podemos encontrar el nexo de la realidad presente y futura, y desde éste punto de vista, habria que unir un compromiso social y político con la totalidad de nuestro estudio. Sin embargo, la reargumentación necesaria para llegar a ello, queda abierta, aunque al parecer sin respuesta.

Michael J. WALKER, en "Analogías oportunes $i$ inoportunes en la investigació prehistòrica: La descomposició del passat» (págs. 63-101), nos plantea como cualquier dato científico supone una hipótesis previa, de donde procede la posible deformación del significado si se aplican componentes intelectuales en base a teorias discutibles -como el difusionismo, opuesto a la evolución autóctona, o el funcionalismo realista, opuesto 
al formalismo simbólico-, en vez de una metodología propia. Pone ejemplos concretos de distorsión, enumerando algunos problemas de interpretación, sobre todo referidos a la prehistoria del sureste peninsular, en los que ha trabajado largo tiempo. Al parecer podria servirnos de consuelo el que los males teóricos de la disciplina no son exclusivos de nuestro pais.

Con gran amenidad y abundante ironia, nos conduce al centro de su exposición: la necesidad de explorar caminos alternativos, mediante programas metodológicos diferentes, con los que abordar los datos, pero sobre todo evitando ambigüedades.

La singularidad de la arqueología no se limitaría a la interpretación global de los restos, sino a que también hay que considerar la representación de las relaciones espaciotemporales, lo que favorece el manejo de un gran número de modelos analógicos, históricos o socioeconómicos. La Prehistoria muestra que la representación del pasado no es una mera imitación del presente etnográfico o el presente histórico, que pueden servir para poner de manifiesto la complejidad del comportamiento humano.

El «sentido común», la intuición y la interpretación imprudente, basadas en analogías no comprobadas, conducirian a un círculo vicioso. En el caso concreto de los inicios del neolítico y su definición, los nuevos paradigmas de las analogias entre la paleoeconomía y la etnologia, han abierto la posibilidad de nuevas orientaciones en los programas de investigación, de cuyo contraste y mayor rigor, puede seguirse criterios metodológicos operativos en distintas direcciones.

Propugna la conveniencia de programas de investigación abiertos y flexibles, frente a los excesivamente rigidos en base a una sóla hipótesis - modelo. El uso de analogías puede ser muy útil en la metodología arqueológica, siempre que se ajusten a los datos arqueológicos y se expresen "en forma de proposición bicondicional», insistiendo en la importancia de que cualquier ensayo de refutación o predicción parta de una hipótesis reducible a una proposición de éste tipo como medida cautelar. De esta forma, la hipótesis de trabajo siempre se aplicaría considerando su posible incapacidad para interpretar todas las observaciones realizadas. El segundo paso sería comprobar la fiabilidad de las predicciones anticipadas, lo que reforzaría la hipótesis previamente contrastada, si es que no ha sido rechazada.

La estrategia de la investigación se basaria asi en "la elección entre hipotesis alternativas y la selección de aquella que pueda aportar los 
mejores criterios de utilidad metodológica". Los modelos resultantes corresponderian a teorias de nivel intermedio.

La singularidad ontológica de la arqueología, que muestra la existencia de cambios, rupturas y desaparición de culturas, cuestiona la conveniencia de aplicar a situaciones pasadas las construcciones teóricas basadas en observaciones actuales. Plantea los posibles problemas de la práctica de un programa "crítico predectible» y su rechazo por Renfrew que no se define por una metodología crítica. El reconocimiento de la singularidad ontológica de la arqueología, exige cautela en la formulación de conjeturas sobre el significado de los restos del pasado. Tras la discusión sobre la conveniencia de formular programas arqueológicos dentro del marco de teorias analógicas, que el autor examina ampliamente, concluye que lo fundamental es dejar de aplicar instrumentos metodológicos ajenos a los datos, tratando de darles significado, considerando en cambio el significado proporcionado por la propia metodología arqueológica. Señala la incongruencia que supone el no utilizar la expresión «conocimiento arqueológico", habitual en otras ciencias, independientemente de sus fuentes de información.

Puede ser útil la elaboración de varios modelos alternativos, incluso incorporando algunas hipótesis de trabajo basadas en observaciones negativas. Es importante diferenciar probabilidad y semejanza, ya que la semejanza del modelo no implica la probabilidad de su incidencia, y la probabilidad con que se puede predecir una serie de observaciones, no supone que haya subyacente un único modelo semejante. Una serie de ejemplos ilustran la aplicación de modelos analógicos y sus resultados.

A pesar de la dimensión de "tiempo distante" de la arqueología, defiende el uso de una metodología crítica y científica, que se esfuerce en refutar hipótesis de trabajo «bicondicionales", cuyos parámetros se tomen de fenómenos del pasado que se investiga, descartando asi las que se vayan excluyendo. En este proceso se debe de tratar de delimitar parámetros definibles que permitan modelos alternativos e interpretaciones generales. En último término, hay que tener en cuenta que la arqueología se ocupa en primer lugar de la cultura material y que la interpretación sociocultural no es el único objetivo.

Los modelos icónicos reducirían la renovación de los datos seleccionados y, es precisamente el proceso de selección el que nos aleja de la realidad. El deseo de inferir la dinámica social a través de la estática de los restos arqueológicos, mediante la transferencia acrítica de determinados planteamientos teóricos, puede llevar a construcciones falseadas que no resisten una comprobación crítica. 
Si los datos son la primera representación de las observaciones arqueológicas, su representación icónica es la segunda. Pero el modelo icónico no indica por sí mismo ningún proceso de transformación, a no ser en lo que se refiere a la lectura que supone la imposición de dirección vectorial, que suele ser arbitraria y condicionada por su adecuación a las observaciones. Además, es muy difícil el cómputo de la probabilidad y verosimilitud de modelos icónicos y la representación de los datos es semilogaritmica y no lineal.

Concluye el autor con una serie de formulaciones y ejemplos en relación con los cambios de la prehistoria. En primer lugar, los modelos a aplicar han de ser multidimensionales, no lineales, y no siempre uniformistas, y sometidos a prueba antes de incorporarlos, debiendo de ser prudentes con las analogías uniformistas. El método crítico y científico, obliga «al perfeccionamiento continuado de la metodología responsable de los datos del pasado y a la confrontación de hipótesis, modelos y teorías dispares".

La ponencia de Peter S. WeLLS, sobre "Models del canvi cultural en la Protohistória europea" (págs. 103-129), se refiere a los cambios producidos durante el I milenio a.C. en Europa -rápido incremento de la actividad económica, desarrollo urbano y formación de unidades políticas, "estados", presididos por grupos de élite social-, comentando los diferentes modelos aplicados a su estudio, bien conocidos en España a través de reciente publicación del autor traducida al castellano.

Durante el Bronce final se manifiesta una aceleración del proceso de cambio económico: incremento de la producción agrícola y de manufacturas de bronce, que son objeto de un activo comercio, así cómo desarrollo de la especialización en la producción de materias básicas y sistemas de intercambio, por parte de determinadas comunidades. En la primera edad del Hierro se van fomando centros comerciales o "ciudades", como en el caso de Hallstatt, centro dedicado a la explotación y comercialización de la sal. Los núcleos de habitación son mayores que los anteriores, de caracter fundamentalmente agricola, y se caracterizan por concentrar actividades artesanales y comerciales. Representan el primer desarrollo urbanístico o de vida ciudadano de la Europa templada, y sus redes comerciales se extienden en todas direcciones hasta alcanzar la Europa mediterránea. En las necrópolis, pequeños grupos de tumbas extraordinariamente ricas, con objetos de lujo importados, parecen indicar un incremento de la estratificación social.

Al comenzar el período de La Tène, parece haber una recesión al norte de los Alpes, aunque en algunas zonas sigue habiendo tumbas 
principescas. El nuevo estilo decorativo que aparece en el Rin medio, podría asociarse a los pueblos celtas o galos de las fuentes antiguas. Se irá produciendo una ruralización de la población de la Europa templada, que se agrupa en pequeñas comunidades autárquicas, disminuyendo la actividad comercial, y relativamente igualitarias, según parece reflejar las necrópolis. A finales del periodo, en los últimos siglos del milenio, se producen cambios importantes hacia una sociedad de carácter urbano, en asentamientos fortificados, los "oppida», a veces de grandes dimensiones, que se convierten en centros económicos y políticos, con una importante producción de hierro, junto a la forja del bronce, y otras actividades artesanales como el vidrio, la madera y el cuero, iniciándose una economía monetaria con acuñaciones propias, empezando también el uso de la escritura. La conquista romana pondrá fin al desarrollo independiente de estas comunidades.

La abundancia de datos arqueológicos en un espacio y tiempo relativamente restringidos, permite abordar la interpretación de los indicios de cambio desde una perspectiva bastante objetiva. El autor comienza haciendo un esquema del tratamiento dado a la cuestión. En una primera etapa, se sobrevaloró el impacto de las culturas mediterráneas sobre las poblaciones indigenas, sin tener en cuenta su propio desarrollo interno, con una complejidad comparable a la de las civilizaciones clásicas. Fenómeno que también se ha dado en el estudio de nuestra Protohistoria peninsular, en que la valoración del peso de las colonizaciones mediterráneas (fenicia o griega, a veces alternativamente, según las modas), o el influjo indoeuropeo, parecian simplificar el problema de la evolución interna de nuestras comunidades indígenas hasta llegar a las formaciones culturales de los pueblos prerromanos. En el caso de los paises escandinavos, la perduración de la edad del Hierro hasta finales de la edad Media, ajenos prácticamente a la romanización, permitió una aproximación más directa a su Protohistoria, aunque siempre se pusiera el énfasis en las influencias exteriores cómo explicación del cambio, y, sobre todo, cómo referencia que permitiera establecer secuencias cronológicas.

La interpretación de los cambios en la Prehistoria europea por Childe, se basó fundamentalmente en los mecanismos difusionistas. Posteriormente, el estudio de la relación entre el hombre y el medio, o las aproximaciones antropológicas a través de la propia dinámica interna de la cultura, relegan a un papel menor las influencias externas, aunque a través de la interrelación comercial contribuyeran a aumentar la complejidad del cambio.

En la actualidad se han adoptado diversas variantes del modelo sistémico, considerando la cultura como un sistema divisible en subsiste- 
mas. El cambio en cualquiera de estos, repercutiría en los demás, siempre que el sistema global restableciera su equilibrio dinámico. Ningún factor individual por sí mismo, puede explicar los cambios culturales, sino por la interacción entre todos ellos. De acuerdo con esto, la investigación se caracteriza por la aceptación de que el cambio es sistémico, aunque se centre en algunos aspectos particulares: cambios en el medio natural, en la tecnología con su repercusión en la economía, la intensificación del comercio y con ello la interrelación entre culturas distintas. Otras veces se ha puesto el énfasis en los cambios de la estructura social, con la aparición de élites, tipos de alianzas y un sistema competitivo. La presión social crearía la necesidad defensiva y el desarrollo de los oppida. En la interacción entre grupos de distinto nivel cultural, lo más importante no sería el comercio, sino el intercambio de ideas. Aunque ya no se hable de migraciones ni de invasiones, parece evidente la importancia de este tipo de contactos.

También hay un nuevo interés por la ideología cómo factor esencial de cambio cultural. Los cambios en creencias religiosas y simbolismo, tendrian un papel principal a comienzos de la segunda edad del Hierro, caracterizada por el estilo de La Tène. A través de la cultura material se comunicaría ideologias concretas cómo medio de establecer y mantener posiciones de autoridad, o para comunicar información sobre creencias e ideas. Mediante la semiótica se ha tratado de extraer información sobre el uso de los signos y símbolos, así cómo sobre su papel en la información dentro de los cambios culturales.

A través de la antropología cultural se ha intentado relacionar las evidencias arqueológicas con los grupos de pueblos (celtas, galos, germanos) mencionados por los autores clásicos, y también la constitución de la identidad cultural de los pueblos germanos y su reflejo en las evidencias arqueológicas. En el fondo es el empleo de una nueva metodología hacia objetivos que ya se plantearon etnólogos y arqueólogos dentro del contexto del nacionalsocialismo, aunque ahora hay que pensar en un interés fundamentalmente científico, sin aquellas connotaciones políticas.

Señala el autor que todas estas orientaciones teóricas no son excluyentes entre sí, sino por el contrario todas contribuyen a una mejor interpretación de la protohistoria europea. El creciente aumento del conocimiento arqueológico, contribuirá, además, mediante un debate informativo y constructivo, a valorar su complejidad y a una mayor comprensión de la naturaleza de los diversos procesos de cambio reflejados en el registro arqueológico. Por mi parte añadiría que esta ponencia es una buena 
muestra de cómo los planteamientos teóricos pueden dar buenos resultados cuando cuentan con una base sólida de conocimiento arqueológico.

Andreu VIOLA RECASENS, se centra en un caso concreto «El collapse maia clàssic i el collapse teòric funcionalista” (págs. 131-143). El manifiesto y rápido colapso de la cultura maya, ha sido objeto de interés y amplio debate en estos últimos tiempos, tomándose incluso como paradigma para interpretar otros casos alejados en el tiempo y el espacio, y el autor nos lo presenta como ejemplo que podria servir para las teorías del cambio en la prehistoria.

El fenómeno conocido como "colapso maya", marcaría la transición entre los periodos clásico y postclásico de las Tierras Bajas, unos $150.000 \mathrm{~km}^{2}$, en el espacio de un siglo (el ix d.C.). Se interrumpe la construcción de edificios, monumentos y estelas, abandonándose los centros ceremoniales y disminuyendo la población sensiblemente, en $1 /$ 2,5 millones. En Tikal concretamente disminuye un 90 por ciento, debido a un aumento de la mortalidad y sobre todo a emigraciones masivas. Desaparecen la agricultura intensiva y el comercio a larga distancia, y se abandonan las actividades relacionadas con el calendario y la escritura jeroglífica, al mismo tiempo que desaparece la élite teocrática. Hay también una gran fragmentación lingüística. La nueva situación muestra un poblamiento en pequeñas aldeas selváticas, igualitarias y relativamente aisladas, que practican una horticultura de subsistencia.

Tras exponer y criticar las distintas interpretaciones funcionalistas catástrofe natural, conquista militar- y sistémicas, valora la importancia de los factores sociales intrínsecos. Señala que sería necesario revisar profundamente las estructuras sociales del periodo clásico, indicando algunas pautas: la progresiva y espectacular diferenciación de la calidad de vida de los campesinos en relación a los miembros de la élite. El malestar social que produce esta diferenciación (disturbios y revueltas documentadas hacia el 530-600). La relacción de las élites regionales, que en el siglo vi se unen para sofocar la rebelión y reforzar la centralización, con resultados nefastos.

En el siglo ix hay una desmembración del poder a favor de señores locales frente a los regionales. Durante el periodo clásico, la concentración de población en los grandes, centros, produjo una creciente dependencia de la importación de alimentos, el monopolio de manufacturas, el control de los pequeños centros situados dentro del área de influencia económica de los grandes, así como la competencia entre los grandes centros regionales. El crecimiento demográfico del periodo clásico final provocó escaseces y carestias y, al mismo tiempo, situaciones de privi- 
legio de las castas dominantes. Las mutilaciones y destrucción de estelas que representaban a los gobernantes, cuyas caras aparecen borradas mientras que las representaciones de los campesinos siempre aparecen intactas, serian testimonio de luchas sociales.

En resumen, la causa del colapso maya, según el autor estaria en las propias contradicciones y desequilibrios socio-económicos internos de la sociedad, mas que en agentes externos que hasta ahora se habian destacado, y cuya influencia, según nos argumenta, debió de ser escasa.

Estas cinco primeras ponencias dedicadas a plantear modelos y factores del fenómeno de cambio en la Prehistoria, fueron objeto de un debate que también recoge el libro (págs. 144-155), iniciándose con el planteamiento de una serie de preguntas por el coordinador, y a las que respondieron los ponentes en sus intervenciones, precisando algunas de sus afirmaciones.

Las tres últimas partes del coloquio corresponden a temas concretos de acuerdo con un orden sincrónico. La comunicación de Eudald CARBONELL, "Variabilitat tecnològica en els objetes d'us dels caçadors recol-lectors de la Prehistòria" (págs. 159-190), se abre con una afirmación que, en los tiempos que corren, podría considerarse sorprendente: "La tecnología es el principal motor de desarrollo espacio-temporal de los homínidos. La tecnología permite la aceleración de estas dos dimensiones". La progresión humana fue lenta. Hasta que la tecnología no alcanzara una alta resolución, no hay una efectiva ocupación de la Tierra por el Homo sapiens. La ocupación del espacio habria estado ligada a la capacidad creciente de "reciclar energía a través de medios endógenos (códigos morfológicos)". La tecnología sería la responsable de la fuerte implantación del hombre, y los objetos de uso los que la asegurarian.

Naturalmente, el autor no aborda el estudio de los útiles desde un punto de vista puramente tipológico, sino a partir de la reconstrucción de la cadena operativa de los útiles, cómo un proceso y un contexto. En breves páginas resume los resultados de años de experimentación y trabajo, aunque en general utiliza una terminología un tanto críptica y complicada para los que no estamos familiarizados con su metodología, lo que a mi modo de ver dificulta el acceso a unos planteamientos sumamente atractivos y hasta convincentes. Trataré de resumirlos.

En la figura 1 ilustra en un esquema el proceso tafonómico, el proceso seguido por los restos hasta su transformación en los elementos de estudio. En este proceso intervienen causas natuales (DYN, dinámica natural) y antrópicas (DYA). Un objeto natural (ON), mediante la intervención de la técnica del hombre prehistórico (DYT), se convierte en un 
objeto tallado (OT). El útil, con una función determinada (OU), puede estar sometido en el yacimiento a modificaciones naturales (DYN), que pueden transformarlo (ONT), hasta convertirse en objeto de estudio (OE) y también ser modificado a causa de su manipulación por los arqueólogos (DYF).

El útil puede adquirir unas dimensiones informativas mediante su estudio a partir de una visión contextual e interactiva. El tratamiento del código informativo del objeto, a través de una teoría general, le permite descubrir el motor dinámico de la estructura morfológica. Esta es sólo uno de los elementos dinámicos del útil, ya que su estructura interactiva se compone de otros elementos sin los que seria imposible comprender los cambios y la evolución de los códigos morfológicos.

Una estructura lítica (útil), se compondria de tres elementos fundamentales: El "morfodinámico", o las formas artificiales producidas por la acción de la técnica, el "morfopotencial», la capacidad de los códigos morfológicos de interaccionar sobre el objeto de trabajo o sobre el medio, $y$ el "morfofuncional", el potencial que ha intervenido o interviene sobre el objeto de trabajo. La relación entre estos elementos es la que hace variar la morfología y operatividad de los objetos de uso. Hay que tener e cuenta también, para entender el útil, la llamada "cadena operativa", es decir, el proceso seguido desde el comienzo de la fabricación del útil hasta su utlización por el hombre sobre el medio y su abandono.

Así, la explicación del cambio de forma del útil, su variabilidad y complejidad, se ha de buscar en la jerarquización y combinación de cada uno de los elementos - morfológico, potencial y funcional-, y en el proceso por el que se produce el llamado efecto transfer. El aprovechamiento de todo el potencial de una asociación lítica, puede dar lugar a la versatilidad de los útiles, es decir, a la transferencia de diferentes posibilidades potenciales y funcionales a una misma estructura morfológica. Con el paso del tiempo, puede variar el espectro lítico, de forma que aparezcan, diacrónicamente, asociaciones de códigos morfológicos de amplia variabilidad, seguidos de otros de pequeño espectro. La versatilidad podria ser la clave de este fenómeno, y, por ello, la transferencia de diferentes valores funcionales a un sólo código morfológico podría ser una de las razones del cambio.

La tecnologia sería la base operativa que ha constituido el medio histórico y sin ella, no podria entenderse la posibilidad que tenian las comunidades prehistóricas de resolver los problemas con que se enfrentaban. La jerarquización de los elementos estructurales le permite establecer los grandes periodos «biotecnológicos»: El periodo «Bio-funcio- 
nal', entre 2,5-1,5 millones de años, en que el Homo habilis y el erectus antiguo serian los creadores de útiles no estandarizados y sin simetria sistemática. El “Bio-morfo-técnico", entre 1,6 millones y 30.000 años, período ya documentado en Europa, cuyos avances (simetria axial y asociaciones de forma piramidal y tiédrica, sistematización del estandar operativo, aumento de la variabilidad morfológica...) se deben a Homo sapiens neandertalensis. El período "Bio-potencial", entre 30.000 y 6.000 años, cuyo autor seria el Homo sapiens sapiens, ya se localiza en todos los continentes y se caracteriza por la introducción sistemática de las formas semitiedras y diedras, con uso de diferentes materiales y morfologías yustapuestas, flexibilización de las láminas, además de la "sistematización de la creoda proyectil" y aparición de los "modelos hidro y aerodinámicos", que mi corto entender no adivina que cosa sean.

Estos grandes periodos tecnológicos, indicarian el paso de estructuras poco complejas a sistemas generales de gran complejidad, que podrian explicarse por las formas de funcionamiento social y estructural, a las que se llegaría a través del útil y su dinámica en el marco de la paleoecología humana.

La reconstrucción paleoecológica a partir de los útiles, se hace en función de la unidad operativa (UO), que seria la asociación de dos impulsos dinámicos (fig. 6), la selección (S) y la interacción (I). El encadenamiento de una cantidad indeterminada de unidades operativas que tengan una misma dirección, da lugar a una tema operativo (TO), el útil. La dinámica de un conjunto de TO da lugar a una estructura operativo (EO), donde se puede entender y captar de forma general, toda una acción dirigida a diferentes objetos de trabajo, pero subordinada a una misma estrategia.

El conjunto de estructuras operativas (EO), daria lugar a la unidad eco-social (UES), marco de referencia de la relación entre el medio natural y el medio histórico. la UES seria la expresión máxima del útil, que ha sido el desencadenante de esta interacción.

Los nuevos métodos analíticos han abierto grandes posibilidades a la definición de los útiles y al conocimiento de su papel en la interacción de las estructuras, y, la reconstrucción de la cadena operativa, marca una nueva línea de investigación. La reconstrucción tecnohistórica, que propugna el autor, es un intento de globalizar y comprender críticamente un proceso de estructura social, a través de los útiles y su interacción en la sociedad y el medio. Sin duda es una tarea dura y difícil, pero desde mi punto de vista refleja una actitud honesta, crítica y realista, dentro de la más estricta investigación arqueológica. Por ello pienso que es una via 
por lo menos tan lícita como las que parten de métodos antropológicoculturales o etnoarqueológicos.

Como era de esperar, esta ponencia dio lugar a un amplio debate. Así, entre otras, la cuestión de la relación genético-cultural entre cambio biológico y tecnológico (J. M. VICENT), la de la sofisticación terminológica ( $F$. CRIADO), la de la utilización de estandars tecnológicos actuales a la determinación de la actividad de instrumentos líticos prehistóricos (Ch. TILLEY), la del intento de periodización tecnológica en relación a las tradicionales fases del Paleolítico ( $R$. MORA), la identificación del cambio tecnológico con el cultural y cual sería el motor del cambio tecnológico (J. PIE), las del carácter ideológico "reaccionario" del punto de partida tecnológico (J. M. VICENT), la de la utilización de la tecnología para definir el caracter humano (C. GAMBLE). Entre las respuestas puntuales del autor, podriamos destacar las de caracter más general. Su justificación al elegir la tecnología como vía de investigación, por considerarla como elemento realmente diferenciador del género humano respecto de los demás, y porque era necesario abordar su estudio de una forma nueva, diferente a las tipologías empírico-tradicionales, sometiéndola a un reciclaje, a una "reconversión industrial». Era necesario volver al material, al registro empírico, con una perspectiva actual, más amplia, explicativa y racional, haciendo converger distintas disciplinas sobre un mismo objetivo.

Clive GamBLE, presentó una ponencia con ei título «La producció alimentaria i els origens de l'agricultura: Una perspectiva caçadora-recolectora" (págs. 197-234), que en realidad no responde del todo a su contenido. Tras una introducción un tanto jocosa sobre la "revolución neolítica", señala que ésta se basa en dos aspectos: el concepto de sociedades complejas y simples, en el que no parece creer demasiado, y en el estudio de su desarrollo centrado en el Próximo Oriente y Europa, con la investigación de la transición a la producción de alimentos, que en cierto modo mediatizaría su estudio. Fuera del espacio geográfico citado, nunca pretendió servir de modelo para el resto del mundo.

El autor en cambio, quiere abordar el estudio de la producción alimentaria fuera de la perspectiva fundamentalmente europea y bajo un aspecto concreto: «la pérdida de la autonomía, asociada a los procesos de producción alimentaria mas que a la aparición de la complejidad y la pretendida propagación de las sociedades igualitarias». Ello se justificaría por la tendencia actual de las ciencias sociales hacia un concepto de ciudadania mundial.

Como ejemplo de esta aproximación global, se refiere a un proyecto dirigido a la investigación del periodo máximo glacial de Würm (Wisconsin 
tardío) en todo el mundo, que reflejaría el impacto relativo de la climatologia glacial en los hemisferios norte y sur y la pervivencia de los cazadores recolectores en Africa meridional y Australia. En general, el ambicioso proyecto no parece que haya aportado grandes novedades.

A continuación, pasa al estudio de la producción alimentaria en Australia, "en un continente donde nadie ha constatado su existencia». Esto sería una ventaja al no tener que revisar trayectorias evolucionistas con objetivos conocidos. Así existe la posibilidad de «examinar la complejidad de las sociedades de cazadores recolectores y adivinar la razón de su autonomia».

El árido desierto del interior del continente se ocupó desde el 22.000 $\mathrm{BP}$, en un periodo de extrema aridez, contrariamente a lo que se creia hasta hace poco, que el centro del continente austral solo fue colonizado a partir del Holoceno. Parece que desde los primeros momentos ya se perfila el marco social básico que caracteriza a los cazadores-recolectores australianos. El sistema de organización territorial estaría basado en la conservación y gestión del conocimiento religioso.

A partir de aquí, resume los resultados de las investigaciones antropológicas sobre grupos de aborígenes. Los derechos sobre la tierra quedan establecidos por lugares sagrados destinados a celebraciones rituales y señalados con representaciones pictóricas. "Estas pinturas rupestres y objetos sagrados, constituyen la propiedad religiosa que se define y asocia a un clan y a los más ancianos de él, incluyendo todos los iniciados". Al parecer, ningún clan puede llegar a ser autónomo, por lo que ha de participar en lazos más amplios para conseguir la reproducción social.

Un cierto número de habitats de las regiones semiáridas del sur de Australia, Tasmania y el norte tropical, indican la temprana aparición del arte prehistórico: anterior al $30.000 \mathrm{BP}$ en las rocas grabadas de Karolta. Pero el principal descubrimiento seria la combinación del arte, los objetos de intercambio y la colonización de los principales ecosistemas australianos durante el Pleistoceno final. Actualmente, los centros rituales asociados al arte reflejan las alianzas interregionales y la autoridad de los mayores en la estructura de los grupos sociales.

Pero este modelo, que parece tener antecedentes tan antiguos, no presenta una continuidad ininterrumpida. Los hallazgos arqueológicos de muchos lugares de Australia indican que los cambios empezaron hace unos cuatro o cinco mil años. Sistemas hidráulicos, selección de nuevas especies vegetales, cambios en la tecnología lítica con aparición de microlitismo, y, sobre todo, la expansión de los asentamientos y el aumento 
del número de hallazgos cerámicos. Estudios sobre esqueletos humanos han revelado que el desarrollo demográfico parece haber sido el factor selectivo de los cambios operados en el Holoceno medio y de la adopción de nuevos recursos.

El interés del ejemplo australiano estaría en que los cambios representan nuevas formas de organización, más complejas que las reveladas a través de modelos ecológicos o hipótesis demográficas, y que los cambios no están en relación con una transformación importante del medio ambiente. En cierto modo, esta afirmación parece una perogrullada, pues ya hace tiempo que se había determinado por deducción, en base a análisis puramente arqueológicos en áreas europeas e incluso del Próximo Oriente, la persistencia de las formas de vida epipaleolíticas en pleno Holoceno, y una tardia aculturación neolítica. Si en realidad lo que pretende el autor es demostrar que el modelo de la "revolución neolítica" no es válido para el caso de Australia, creo que no valía la pena tanto esfuerzo, seguramente es así, pero lo más probable es que tampoco haya ningún otro modelo aplicable, ni mucho menos, a nivel mundial.

Asi, cuando pasa a tratar el caso de Europa, se refiere al modelo de "aprovechamiento de recursos", frente al de difusión. "El modelo de aprovechamiento tiene unas fronteras muy amplias (de hecho, una zona entera de contacto recolector/agricultor). Esto implica un sistema mundial dentro del cual la transformación de las estructuras existentes no fue adoptada uniformemente, y dentro del cual los procesos fueron más puntuales que graduales en el tiempo". La clave de este modelo estaria en los "cazadores complejos" de las costas atlánticas, cuyo rasgo principal seria el sedentarismo, en oposición a la migración estacional. De acuerdo con este modelo, la agricultura estaría representada por una serie de rasgos definidos (trigo, cebada, oveja, cabra, cerdo, buey), junto con una reducción o eliminación de determinados recursos no domesticables. Pero este modelo no aborda la cuestión de la reproducción, la estructura social o la "pérdida de la autonomía", que es lo que le interesa, y alguno de sus rasgos podría remontarse al Pleistoceno, además parece muy dificil definir que son los cazadores complejos.

Finalmente, trata de la "pérdida de la autonomía" cómo modelo de desarrollo, que, al parecer, era el objetivo de la ponencia, al que ha precedido tan largo discurso. Entre los cazadores recolectores habría una continua demanda entre la organización del trabajo para la explotación de recursos y la necesidad de sistemas de alianzas entre grupos, a través de matrimonios e intercambios, en los que pudo surgir el principio de competitividad. A continuación presenta una serie de hipótesis sobre los sistemas de explotación y sus objetivos, como consecuencia de un incre- 
mento de la demanda, y las diversas estrategias de los cazadores: mobilidad, costes, productividad, reducción de las áreas de recolección, y, como consecuencia, disminución de los campamentos móbiles, lo que tenderia a una mayor sedentarización. De ahí se pasa a "la opción de investir de autoridad a un representante del grupo", encargado de negociar con otros grupos cuando hubiera conflicto de intereses. Esta autoridad inicial iria revistiéndose de nuevas atribuciones y un estatus especial: control y conocimiento de símbolos, rituales y creencias. Todo ello seria consecuencia del deseo de incrementar la producción local.

El papel del "negotiator" resolvería en parte el problema, pero "representó una pérdida de autonomía a nivel de participación directa». Había que mantener al "negotiator" para que tuviera libertad de acción. Se desarrollaron nuevos y más eficaces sistemas de producción: irrigación bancales, domesticación. La pérdida de autonomía a nivel doméstico y local se compensaba con la posibilidad de mejorar la estructura de competición en el sistema de alianzas. "La creación de papeles sociales diferenciados presentaría enormes posibilidades para el cambio, con muchas direcciones posibles".

Según el autor, habria que separar la cuestión de la producción de alimentos de la de complejidad social y posterior estratificación, aunque fuera "una condición previa necesaria pero no suficiente para su aparición". La división entre cazadores y agricultores basada en los rasgos establecidos por la "revolución neolítica" no es suficiente, ni tampoco para hacer una distinción entre cazadores complejos y simples. Reconoce el autor que las sociedades cazadoras recolectoras están en continuo cambio, y, que "desde una perspectiva prehistórica, esto significa que, probablemente, tendríamos que incluir, si la distinción económica tiene un valor permanente, rasgos de las sociedades del neolítico europeo antiguo y medio". La pérdida de la autonomía se produciría a pequeña escala y no "a una escala integrada dentro de la sociedad compleja europea".

Todas estas ingeniosas reflexiones - cómo en su dia lo fueron las de los misioneros megalíticos o los colonos orientales-, nos han llevado, por mano del autor, de Austria a África, a América y a Europa, pero dejando sin resolver la explicación de la experimentación sobre los recursos que condujo a la domesticación y de su papel en la evolución y complejidad de las sociedades. El clima, la población, la tecnología y la buena suerte, serian razones poco convincentes por si mismas. El autor sugiere que la aparición y la proliferación del arte, adorno, instrumentos musicales y objetos rituales, que se han considerado reflejo del desarrollo de la complejidad sociocultural, si se considera en relación a su distribución espacial, se limita a las latitudes septentrionales. De nuevo hace un 
recorrido por los cinco continentes, y aventura "que no es ninguna coincidencia, que la aparición de la agricultura y la domesticación de animales, se produzca antes en aquellas áreas donde los sistemas territoriales, inferidos a partir de la distribución del arte rupestre, fueron quizas más débiles, y que los nuevos recursos y pautas de explotación seguramente serian introducidos en las áreas meridionales y septentrionales, en las que las pinturas y elementos ornamentales eran más comunes".

En fin, tras rizar tantos rizos, el autor concluye que la aparición inicial de la producción de alimentos, no supuso un corte en la Prehistoria «sino una continua intensificación, en algunas regiones, de las prácticas socioeconómicas proporcionadas por la existencia de una ideologia y una estructura social definidas". Es curioso que, ante todas estas "especulaciones deliberadas" - reconocidas por el autor-, en el capítulo de debate no hubiera ninguna intervención en torno a la metodología, o más bien a su ausencia. Por lo visto se acogieron complacidamente su «desafío a algunas visiones tradicionales" y su caótico viaje por el universo prehistórico, en el que no se hasta que punto ha podido pesar la traducción que tengo entre las manos.

Las dos últimas ponencias están dedicadas al Neolítico. Juan Manuel VICENT, en su exposición sobre «El Neolític: Transformacions socials i econòmiques" (págs. 241-293), vuelve a plantear, desde una perspectiva marxista de sociedad de clases, el concepto de "revolución neolitica", "una de aquellas ideas clave cuya evolución conceptual permite reconstruir claramente la historia conceptual de una disciplina, en éste caso la Prehistoria», frente al materialismo ecológico como "pardigma dominante's.

Pero sobre todo hay que tener en cuenta el punto de partida del autor, ya conocido en otros escritos suyos, su concepto de Prehistoria y Arqueologia. La Prehistoria sería el "proceso general de producción del conocimiento histórico", mientras que la Arqueología sería simplemente "la elaboración y la interpretación del registro arqueológico". Esta dicotomia, esta separación entre Prehistoria y Arqueología Prehistórica, desde mi punto de vista, puede ser causa de fuertes limitaciones a la hora de llegar a una interpretación histórica de la Prehistoria. Es como si un historiador de la antigüedad se limitara a consultar las fuentes a través de buenas ediciones a cargo de filólogos o epigrafistas, sin contrastar personalmente los textos antiguos, o cómo si un medievalista no fuera también paleógrafo ni conociera el latín, el árabe o el hebreo, según los casos, para poder profundizar en el contenido y estructura de las fuentes. 
El acceder a la información arqueológica a través de manuales y obras de sintesis, supone, en primer lugar, aceptar sin reservas la "auctoritas" del arqueólogo-prehistoriador, que muchas veces parte de planteamientos metodológicos distintos, $y$, en segundo, aceptar el criterio sintético y reduccionista del autor, su valoración de lo más importante. Aunque pueda resultar cómodo acceder inicialmente a una información de síntesis actualizada, es necesario recurrir y comprobar críticamente los datos en las monografías que han servido de base, sobre todo, teniendo en cuenta que, a juicio del autor, "La investigación española actual, tanto la orientada por los tradicionales puntos de vista morfo-tipológicos e histórico-culturales, como la que se comienza a practicar desde planteamientos ecológicos-culturales y/o socio-económicos, acepta como punto de partida indiscutible la periodización tradicional».

Asi cuando generaliza la continuidad arqueológica Epipaleolítico-Neolítico antiguo, que tanto se ha investigado estos últimos años, precisamente con el objetivo de "demostrar" un origen local a nuestro neolítico, solo se ha podido comprobar en determinados casos en que es evidente la aculturación de poblaciones epipaleolíticas. En otros no parece quedar comprobada ésta continuidad. Bastaría leer la monografía dedicada a la Coveta de l'Or, para ver que las cosas no son tan simples. Naturalmente, en lo que si estamos de acuerdo es en que lo importante, lo que debe buscar el prehistoriador, no es simplemente una serie de datos sino la interpretación total del contexto, que en palabras del autor sería «la estrategia de producción en la que intervienen y el tipo de relaciones sociales que ésta estrategia genera". Precisamente las elaboraciones teóricas de Childe fueron posibles porque tenían una sólida base arqueológica. No era un simple teorizador, sino ante todo fue un buen arqueólogo, con un conocimiento poco frecuente de un amplio espacio prehistórico.

Señala el autor la importancia de las nuevas aportaciones del arte postpaleolítico y la necesidad de incorporar su estudio al proceso inicial del neolítico, aunque no se plantee su relación con los conceptos de continuidad y cambio. En todo caso, cómo en ciertas interpretaciones del arte paleolítico, sería indicador de la aparición de una conciencia territorial, cómo un sistema primitivo de apropiación de la tierra, e indicaría la existencia de un orden simbólico elaborado que afecta a diversas esferas de la vida social. La decoración cerámica, los adornos, indicarian que ya en el $\vee$ milenio existía la necesidad de expesar simbólicamente un cierto número de relaciones sociales, y a ello se dedicaba una parte del trabajo social.

La existencia de lagunas en la investigación hace muy dificil aceptar la periodización establecida en base a determinadas secuencias regio- 
nales, que se aplican a otras zonas por simples referencias tipológicas y que es consecuencia de grandes desequilibrios en el conocimiento. Pienso que, de momento, no nos podemos permitir grandes generalizaciones y que la fragmentación regional de que se lamenta el autor, en todo caso puede ser un primer paso necesario y de cautela, ante una evidente falta de información. Precisamente su indicación de que en el IV milenio es imposible aplicar una periodización tradicional -neolítico medio-, es una buena prueba del complejo y vario desarrollo de nuestras comunidades neolíticas en un momento sincrónico, con manifestaciones totalmente opuestas a la concepción de un evolucionismo lineal. No creo que sea un fallo en el registro, sino más bien que se trata de un período de cambios y diversificación, cada vez más patentes, conforme aumenta nuestro conocimiento.

La instalación en poblados al aire libre, por lo menos desde el IV milenio, es uno de los temas de mayor interés en la investigación actual del Neolítico peninsular, que permitirá una mejor aproximación a la organización interna de éstas comunidades y a sus estrategias económicas y territoriales, con toda la problemática que encierra la conservación de éste tipo de yacimientos. Su consolidación en poblados "de larga ocupación", en algunos casos, no siempre, es uno de los rasgos de la transición del Neolítico final al Calcolítico. La continuidad entre ellos, tendría un punto de inflexión en la ampliación del intercambio de materiales a larga distancia, y sería en éste contexto donde habría que situar el comienzo de la metalurgia.

Insistiendo en el problema de la periodización, señala a modo de ejemplo, tres horizontes arqueológicos «interculturales", la cardial, el megalitismo y el campaniforme, que, evidentemente, se escapan de un esquema rígido en espacio y tiempo, que no corresponde a la realidad del estado actual de conocimiento. Su actitud crítica hacia la secuencia tradicional, parte de las mismas anomalias que presenta, y que, según el autor, se deben a la resistencia del proceso histórico a ser fragmentado ya que la continuidad histórica aparece enmascarada por la segmentación arqueológica.

En realidad, las distintas periodizaciones, no son más que un sistema más o menos práctico, o cómodo, de ordenar una secuencia continuada. No creo que esté en el ánimo de nadie el establecer cortes rotundos, artificiales, cómo puedan serlo los que en su día se hicieron para "dividir" la Historia antigua, media, moderna y contemporánea, simplemente por cuestiones de orden práctico. La labor del prehistoriador consiste precisamente en explicar cuando, como y porqué se produce un 
proceso de cambio, y cuales son los presupuestos en que se basa su explicación.

En este caso, el autor no parece admitir un cambio epipaleolítico/ neolítico antiguo, en que la presencia de especies domésticas, quedaría dentro de una estrategia de "amplio espectro", característica del mesolítico y su búsqueda de estabilidad en un medio inseguro. Los primeros ensayos agricolas o ganaderos no supondrian el inicio de una economia productora, "sino la progresiva transformación de las economías de caza y recolección en sistemas de consumo actualizado, resultado de un esfuerzo de estabilización a largo plazo de las frágiles condiciones ecológicas del Mediterráneo".

Los procesos de cambio perceptibles en el registro arqueológico corresponderian a lo que denomina "hiatus del IV milenio", en que se produciria una transformación radical, aunque gradual, de una gran parte de las poblaciones de la Península, y al que podría aplicarse el término «revolución», entendido cómo revolución social, no cómo un cambio en la economía, sino como una transformación de la "economía política". Sería el «punto de inflexión» indicador del fin de las sociedades propiamente "primitivas" y el comienzo de un nuevo ciclo histórico, el de las sociedades "campesinas", que implica la creación de nuevas formas de organización social y "a la larga, modifica el destino histórico de la humanidad".

Estoy de acuerdo en que en el IV milenio, el registro arqueológico va precisando el resultado de los cambios iniciados mucho antes en determinados lugares de la Península. En estos mil años, la dispersión del poblamiento y los caracteres de los asentamientos parecen indicar una auténtica colonización de tierras de muy distintas posibilidades económicas, lo que, lógicamente, supone diversas estrategias de ocupación y organización interna de los grupos. Me parece dificil establecer un único modelo válido para todos ellos a lo largo de tan dilatado período.

El modo de vida campesino, que estaría caracterizado por su vinculación directa y permanente a la tierra y sus orígenes habria que buscarlos en los «esquemas genealógicos de apropiación». El linaje ofrecería el marco de unas relaciones de producción estables que garantizaran el acceso exclusivo del grupo «al trabajo social inmovilizado en sus medios de producción" y "la reproducción del grupo como poseedor de estos medios". En este sentido, campesino no quiere decir necesariamente agricultor, cómo en los "pagesos del Pla" en la denominación de Tarradell a las gentes de los sepulcros de fosa. Lo difícil es saber cuando realmente nos encontramos ante una sociedad de linajes, que no nece- 
sariamente tiene que estar vinculada a formas de vida neolíticas. El problema de esta arqueología social está precisamente en su correspondencia con la documentación arqueológica. El enterramiento colectivo sería expresión de grupos locales de filiación, y la reiteración del uso de un mismo lugar funerario, expresaria la ocupación permanente del territorio. El trabajo social empleado en la construcción de monumentos funerarios, formaria parte del sistema de reproducción del grupo. Siguiendo a Felipe Criado, piensa que el megalitismo define por primera vez en muchas regiones un paisaje agrario estable, en contraposición al paisaje abierto anterior. Habría que preguntarse porqué durante el IV milenio se dan también otro tipo de enterramientos, concretamente debajo de las viviendas, si esto no significa una apropiación permanente del territorio y una vinculación a un linaje o a una familia, la que ocupa la casa, cómo ocurre en el caso de los sepulcros de fosa.

Josep Maria MiRo y Josep BosCH, en su ponencia sobre «El procés de neolitització a Catalunya. Proposta de desenvolupament de la Teoría de l'Aculturació" (págs. 295-330), hacen una clara introducción metodológica, que es un excelente punto de partida al tema que proponen. Comparto su actitud "lógico-histórica", para llegar a una adecuada interpretación. El problema está en que el interrogador esté en posesión de una lógica histórica, y, sobre todo, en precisar si es posible aplicar una lógica formal a la interpretación histórico-arqueológica cómo estructura del razonamiento deductivo.

La aplicación de una lógica empírica por el método de inducción sobre los datos arqueológicos, aislando las relaciones de causalidad, podria llevar, teóricamente, por deducción, a la explicación de los hechos. Pero la explicación de la lógica formal, bien sea cómo un sistema de reglas para deducir a partir de ellas, o, lo que es lo mismo, mediante la afirmación de teoremas cuya aplicación dará lugar a una regla derivada, presenta muchos problemas. En primer lugar, sería necesario disponer de axiomas y unas cuantas reglas, para derivar de ellas otros teoremas, en una operación de derivación mecánica, de transformación de fórmulas mediante la aplicación de reglas. Se trata de un principio teórico que está sujeto a muchas limitaciones, aunque su justificación sea la búsqueda de una garantía de exactitud y objetividad máximas, y la eliminación en principio del error debido a las insuficiencias de la intuición. En este sentido, el uso de formalismos lógicos en el cálculo de enunciados, y, sobre todo de predicados, puede dar buenos resultados, en particular en el estudio de las relaciones formales, una de las carencias más importantes de la lógica tradicional. 
Pero reconociendo que, honestamente, tenemos que sustituir axiomas y reglas por hipótesis y modelos, siempre la respuesta tendrá que darla el acto empírico, y esta es la causa en último término de la caducidad de las hipótesis y modelos explicativos, que, sin embargo, han servido para que se podujeran notables avances en nuestra investigación prehistórica.

Los autores desarrollan el modelo teórico aculturacionista como vía de interpretación del proceso de neolitización en Cataluña, que en su formulación actual presenta contradiciones: aunque se acepte la importancia del papel de las comunidades epipaleoliticas, el detonante del proceso de neolitización se atribuye a estímulos, contactos o préstamos culturales foráneos, y además, el paso del Epipaleolítico al Neolítico se ha enfocado desde un punto de vista estrictamente cronológico, de acuerdo con una visión lineal diacrónica, en detrimento del enfoque espacial sincrónico. Ellos proponen incidir en la propia dinámica interna de transformación de las sociedades epipaleolíticas y en la determinación de las interacciones entre comunidades culturalmente diferenciadas, teniendo en cuenta que la teoria de la aculturación parte de la aceptación explícita de contactos en el espacio y el tiempo, entre sociedades con diferentes niveles socio económicos. En este sentido, sería de gran utilidad el concepto de «frontera» para contribuir a la explicación del cambio cultural en el Mediterráneo occidental.

Al llegar a este punto, preguntaria si la neolitización de Cataluña responde siempre a un fenómeno de aculturación, y en este caso, cuales son los diferentes niveles socioeconómicos que han entrado en interacción, ¿hay alguno que pueda considerarse ya neolítico o con una dinámica interna suficiente para considerarla cómo posible motor de cambio, aunque sea al otro lado de una frontera? En el problema de porqué en algunos casos la interacción tiene poca incidencia en algunas comunidades, mientras en otras llega a transformarlas, habria que considerar hasta que punto valia la pena, o era necesario y rentable, en uno y otro caso, el esfuerzo del trabajo de producción que supone la nueva economía y, sobre todo, la readaptación de los esquemas sociales ya establecidos. Ya que, cómo señalan los autores, la neolitización supone una modificación de las pautas culturales y la incorporación de nuevas formas de producción y nuevas tecnologias, y actualmente sabemos que muchas de estas comunidades epipaleolíticas eran autosuficientes y parecen conservar un gran equilibrio socioeconómico durante largo tiempo.

La receptividad y la dinámica activa de las interacciones serian, a su juicio, la explicación del proceso. El crecimiento demográfico, el incremento cuantitativo y cualitativo de los recursos potenciales, el desarrollo 
tecno-económico, la reducción del territorio a explotar, el desequilibrio entre demanda y capacidad de producción, podrían indicar orientaciones a la investigación para establecer "las variables causales de la intensificación de la producción en las sociedades epipaleolíticas".

En Cataluña y zonas próximas, hay pocos datos en el registro arqueológico referidos a tecnología lítica y explotación de recursos, por lo que los autores inciden en las estrategias de subsistencia para derivar su predisposición a aceptar las nuevas formas de vida. Tras analizar el estado de la cuestión en relación a una posible domesticación local y su problemática, piensan que las comunidades establecieron una relación más estrecha con las especies explotadas a nivel de caza selectiva o control de excedente de los rebaños. Señalan una cierta diversificación en la explotación de especies, matizada en relación con las variables cronológicas y espaciales, $y$, sobre todo, el predominio de la cabra pirenaica en relación al ciervo.

La posibilidad de una agricultura autóctona, también parece ser negativa, pero si se puede constatar una explotación intensiva de recursos vegetales, en lo que se ha llamado una protohorticultura mesolítica de leguminosas y frutos. Es importante también el aprovechamiento de recursos acuáticos y su posible tratamiento para almacenaje. Todo ello reflejaría una intensificación de la producción de recursos con un criterio selectivo, con una directa incidencia en el entorno, lo que podría indicar que ya se estaban desarrollando algunas pautas, tradicionalmente relacionadas con la economia neolítica, cómo el almacenaje de alimentos.

El concepto de "frontera agrícola", basado en los excedentes demográficos y la superioridad tecnológica aplicada sistemáticamente sobre las poblaciones indígenas, no parece poder aplicarse a la expansión de la agricultura en el Mediterráneo, inclinándose los autores por modelos de interacciones entre cazadores y agricultores: "la adquisición y asimilación gradual de las técnicas y recursos de la economía de producción por parte de las comunidades epipaleolíticas, que adaptarian estas innovaciones a sus necesidades y estrategias de subsistencia". El hecho de ésta adopción gradual y selectiva, sería un claro exponente del fenómeno de aculturación. En bastantes ocasiones el registro arqueológico-cerámicas en conexión con industria lítica de tipo epipaleolítico- confirmaria este proceso. La existencia de una dualidad técnica lítica, con clara diferenciación estructural entre complejos epipaleoliticos y neoliticos, y al mismo tiempo, sus interferencias, podrian indicar también contactos culturales entre ambos grupos, lo mismo que la presencia de ovicápridos domésticos en contextos del epipaleolítico final. 
La aculturación de rasgos de economía neolítica por parte de comunidades de cazadores-recolectores, sería así el resultado de interacciones entre comunidades culturalmente diferenciadas. Esto sería posible porque las comunidades epipaleolíticas "ya habían desarrollado estrategias de intensificación dentro de su propio sistema económico". Las interacciones tendrían así un papel activo en el cambio cultural del Mediterráneo occidental a lo largo de los milenios $\mathrm{VI}$ y $\mathrm{V}$. Los autores no abordan el problema de la presencia de la economía neolítica en el Mediterráneo occidental, a partir de la cual fueron posibles estos fenómenos de aculturación, ya que en esta ocasión no entraba dentro de sus objetivos. 